\title{
LETTER \\ Dynamic Forest: An Efficient Index Structure for NAND Flash Memory
}

\author{
Chul-Woong YANG ${ }^{\dagger}$, Member, Ki YONG LEE ${ }^{\dagger \text { a) }}$, Myoung HO KIM ${ }^{\dagger}$, and Yoon-Joon $\mathrm{LEE}^{\dagger}$, Nonmembers
}

SUMMARY In this paper, we present an efficient index structure for NAND flash memory, called the Dynamic Forest (D-Forest). Since write operations incur high overhead on NAND flash memory, D-Forest is designed to minimize write operations for index updates. The experimental results show that D-Forest significantly reduces write operations compared to the conventional B+-tree.

key words: NAND flash memory, D-Forest, index structure

\section{Introduction}

NAND flash memory is becoming widely adopted as storage media for many mobile devices. However, write operations incur high overhead on NAND flash memory since it has following characteristics [1]: (1) A write operation is 5-10 times slower than a read operation. (2) A write operation consumes 5-10 times more energy than a read operation. (3) Overwriting a page must be preceded by a block erase operation, which is even much slower than a write operation. (4) The number of write-erase cycles for a block is limited to $10,000-100,000$. Therefore, it is important to reduce write operations (and erase operations as well) to improve overall system performance and the lifetime of NAND flash memory. As the volume of data stored in flash memory increases, the use of embedded database systems on flash memory is rapidly increasing. In order to quickly retrieve requested data from a large amount of data, database systems or file systems require efficient index structures. In this paper, we propose an efficient index structure for NAND flash memory, called the Dynamic Forest (D-Forest). By minimizing write operations for index updates, D-Forest can reduce the latency and energy consumption of a mobile device equipped with NAND flash memory.

\section{Motivation}

The $\mathrm{B}+$-tree is one of the most popular index structures in database systems [2]. Although the $\mathrm{B}+$-tree has been successfully used in disk-based systems, it suffers from serious problems in NAND flash memory. Consider a B+-tree with seven nodes in Fig. 1 (a). Assume that each node of a $\mathrm{B}+$-tree is stored in a page of NAND flash memory. Since in-place updates are not allowed in NAND flash memory, if

\footnotetext{
Manuscript received December 24, 2008.

${ }^{\dagger}$ The authors are with the Department of Computer Science, KAIST, Daejeon, Korea.

a)E-mail: kylee@dbserver.kaist.ac.kr (Corresponding author) DOI: 10.1587/transinf.E92.D.1181
}

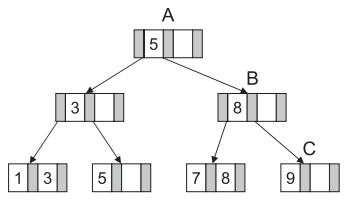

(a) Example B+-tree

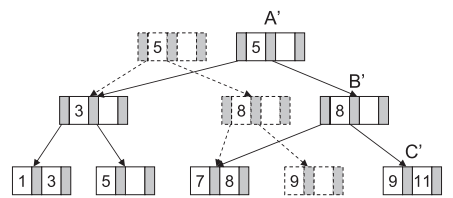

(b) Example of B+-tree up date
Fig. 1 B +-tree on NAND flash memory.

node $C$ is to be updated by insertion of a key, i.e., 11 , the updated $C$, denoted by $C^{\prime}$, needs to be written to another empty page that has been erased in advance. Furthermore, since the physical location of $C$ has been changed, node $B$ that has the address of node $C$ also needs to be updated and written to another empty page. The same procedure continues until the root node $A$ is updated to $A^{\prime}$ and written into another page, as illustrated in Fig. 1 (b). Thus, even a single update to a node can cause many page writes. To make matters worse, if the leaf node is full before insertion or has too small number of keys after deletion, the node split or merge operations are performed throughout several nodes to balance the tree, resulting in even more page writes. There are many strategies for allocating empty pages for page writes [1]. However, the above problem arises in any case when the nodes of a B+-tree have the physical addresses of other nodes.

\section{Dynamic Forest}

\subsection{Overview}

Suppose that a B+-tree $T$ is stored in NAND flash memory. Since it is very expensive to write updated nodes into flash memory on each index update, we maintain a small B+-tree $T^{R}$ in RAM. Let $M$ be the maximum allowed size for $T^{R}$. When a key is inserted, instead of updating $T$ stored in flash memory, we insert the key into $T^{R}$. Similarly, when a key is deleted, we insert the key into $T^{R}$ with a deletion mark. When an inserted key in $T^{R}$ is deleted, we delete the key from $T^{R}$. When a key is looked up, we first search $T^{R}$. If the key is not found in $T^{R}$, then we search $T$. The use of $T^{R}$ allows us to coalesce many page updates into far fewer page writes, as will be described below.

As keys are inserted and deleted, $T^{R}$ grows. When the size of $T^{R}$ reaches $M$, we propagate the changes held in $T^{R}$ to flash memory. In order to propagate the changes held in $T^{R}$ to flash memory, we can perform one of the following operations, depending on the relative size of $T^{R}$ and $T$ : 
- Merge: We merge $T_{R}$ and $T$ into a new $\mathrm{B}+$-tree $T^{\prime}$ and write $T^{\prime}$ into flash memory. By merging, we mean that we construct a completely new $\mathrm{B}+$-tree $T^{\prime}$ from scratch that contains all keys in $T^{R}$ and $T$. (The detail of this merge operation will be described in Sect. 3.3.) Then, $T$ is invalidated in flash memory. As a result, we have only one $\mathrm{B}+$-tree $T^{\prime}$ in flash memory.

- No Merge: We just write $T^{R}$ into flash memory as another B+-tree $T^{\prime}$ without touching $T$. As a result, we have two $\mathrm{B}+$-trees $T$ and $T^{\prime}$ in flash memory.

Although the merge operation creates a completely new B+tree from keys in $T^{R}$ and $T$, it can be performed very efficiently, as will be described in Sect. 3.3.

If we choose to perform No Merge, i.e., we do not merge $T^{R}$ and $T$, the number of page writes is minimized but the search performance of the index degrades because we have to search two trees $T$ and $T^{\prime}$ in the worst case. Furthermore, if we do not merge $T^{R}$ and $\mathrm{B}+$-trees stored in flash memory as more keys are inserted or deleted, the number of $\mathrm{B}$--trees in flash memory increases, resulting in poorer search performance. On the other hand, if we merge $T_{R}$ and $T$ every time the size of $T^{R}$ reaches $M$, although the number of $\mathrm{B}+$-trees in flash memory is minimized to 1 , it may cause a significant number of page writes. Therefore, we need an effective merge strategy to reduce the number of page writes while providing acceptable search performance. In the next section, we describe the proposed index structure, D-Forest.

\subsection{Construction of D-Forest}

Let $|T|$ be the size of a $\mathrm{B}+-$ tree $T$. Our proposed index structure, called the D-Forest, consists of a set of B+-trees with different sizes:

$$
D F=\left\{T_{k} \mid k \in\{0,1,2, \ldots\}\right\}
$$

where $T_{k}$ is a $\mathrm{B}+$-tree stored in flash memory whose size satisfies the following condition:

$$
\begin{aligned}
0 & <\left|T_{0}\right| \leq M \\
2^{k-1} \times M & <\left|T_{k}\right| \leq 2^{k} \times M, \text { for } k=1,2, \ldots
\end{aligned}
$$

Here, $k$ is called the order of $T_{k}$. When $\left|T^{R}\right|$ reaches $M$, we merge $T^{R}$ and the following set of trees in $D F$ into a new tree $T_{\text {new }}$ :

$$
S=\left\{T_{k} \mid T_{k} \in D F, 0 \leq k<m\right\}
$$

where $m$ is the lowest order such that $T_{m} \notin D F$. For example, when $D F=\left\{T_{0}, T_{1}, T_{2}, T_{4}, T_{7}\right\}$, if $\left|T^{R}\right|$ reaches $M$, we merge $T^{R}$ and $S=\left\{T_{0}, T_{1}, T_{2}\right\}$ into $T_{\text {new }}$ since $T_{3} \notin D F$. When $D F=\emptyset$, we just write $T^{R}$ into flash memory as $T_{0}$. Note that the order $k$ of $T_{\text {new }}$ is determined by $\left|T_{\text {new }}\right|$ according to the above condition $(0 \leq k \leq m)$. As a result of the merge operation, $T_{n e w}$ is written sequentially at the end of the index. Then, all trees in $S$ is invalidated in flash memory and $T^{R}$ is removed from RAM.

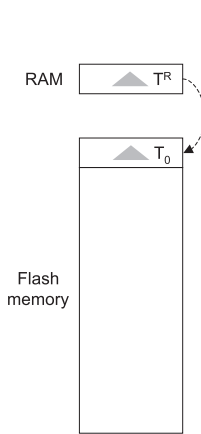

(a)

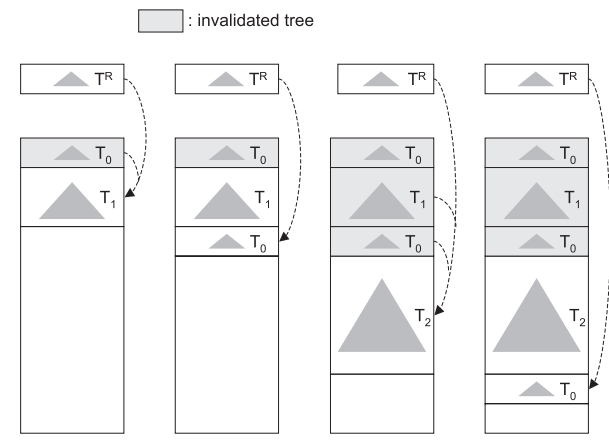

(c) (d) (e)
Fig. 2 Example of construction of a D-Forest.

Figure 2 shows an example of construction of a DForest for each time $\left|T^{R}\right|$ reaches $M$. In Fig. 2 (a), since $D F=\emptyset, T^{R}$ is written into flash memory as $T_{0}$. In Fig. 2 (b), since $T_{1} \notin D F, T^{R}$ is merged with $\left\{T_{0}\right\}$ into $T_{1}$ and $T_{1}$ is written into flash memory. Then, $T_{0}$ is invalidated. In Fig. 2 (c), since $T_{0} \notin D F, T^{R}$ is written into flash memory as $T_{0}$. In Fig. $2(\mathrm{~d})$, since $T_{2} \notin D F, T^{R}$ is merged with $\left\{T_{0}, T_{1}\right\}$ into $T_{2}$ and $T_{2}$ is written into flash memory. Then, $T_{0}$ and $T_{1}$ are invalidated. Finally, in Fig. 2 (e), since $T_{0} \notin D F, T^{R}$ is written into flash memory as $T_{0}$.

For each tree $T$, we keep track of the number of keys that have deletion marks, $D(T)$, and that do not have deletion marks, $I(T)$. The total number of keys in $T$, denoted by $N(T)$, is then $N(T)=D(T)+I(T)$. Whenever $\left|T^{R}\right|$ reaches $M$, we check whether the following condition holds:

$$
\sum_{T \in H} I(T)-\sum_{T \in H} D(T) \geq \frac{1}{2} \cdot \sum_{T \in H} N(T),
$$

where $H=\left\{T^{R}\right\} \cup D F$. Especially when $\left|T^{R}\right|$ reaches $M$ and the above condition does not hold, we merge $T^{R}$ and all trees in $D F$ into only one single tree $T_{n e w}$ in $D F$. As a result of this merge operation, all keys that have deletion marks are removed from $T_{n e w}$, as will be described in detail in the next subsection. Otherwise, we merge $T^{R}$ and only trees in $S$ as described above. This prevents the number of valid keys, i.e., non-deleted keys, in $D F$ from falling below half the total number of keys in $D F$.

\subsection{Merge Operation}

In this subsection, we describe the merge operation in detail. Suppose that we merge $T^{R}$ and a set of $\mathrm{B}+$-tree $\left\{T_{0}, T_{1}, \ldots\right.$, $\left.T_{m-1}\right\}$ into $T_{\text {new }}$. As mentioned in Sect. 3.1, this means that we construct a completely new $\mathrm{B}+$-tree $T_{\text {new }}$ from scratch that contains all keys in $T^{R}$ and $\left\{T_{0}, T_{1}, \ldots, T_{m-1}\right\}$. Efficient construction of a $\mathrm{B}+$-tree from a given set of keys has already been investigated [3]. In our paper, we use an algorithm similar to that proposed in [3]. Figure 3 shows an example of merging two $\mathrm{B}+$-trees. Note that a $\mathrm{B}+$-tree keeps all keys in sorted order in its leaf nodes (Fig. 3 (a)). In this algorithm, we construct the leaf nodes of $T_{\text {new }}$ by scanning and paginating the sorted keys in the leaf nodes of B+-trees 
$1,3,5,7,9 \quad 2,4,6,8,10$

(a)

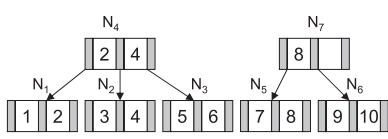

(c)

Example of the merge operation. to be merged. Whenever a leaf node of $T_{\text {new }}$ is constructed, we form an entry corresponding to this node and then hand it over to the first internal level. Similarly, when a node of the first internal level is constructed, we also form an entry corresponding to this node and then hand it over to its next higher level. We repeat this process until all the keys in the leaf nodes of B+-trees to be merged are paginated. For example, in Fig. 3 (b), after keys 1, 2, ., 6 are paginated, node $N_{1}, N_{2}$, and $N_{3}$ in the leaf level are constructed and then $N_{4}$ in the first internal level is constructed. In Fig. 3 (c), after keys 7, 8, 9, 10 are paginated, $N_{5}$ and $N_{6}$ in the leaf level are constructed and then $N_{7}$ in the first internal level is constructed. After that, in Fig. 3 (d), $N_{8}$ in the second internal level, i.e., the root node, is constructed. When we construct each page, we store the maximum number of entries in each page without leaving extra storage space. This makes D-Forest maximize storage utilization and, as a result, the number of page writes required to store the merged $\mathrm{B}+$-tree is minimized. Note that $\mathrm{B}+$-trees stored in flash memory are never updated in D-Forest. Therefore, the merge operation can be performed in $O(N)$ time, where $N$ is the number of keys. More importantly, this merge operation requires no additional write operations, except for writing the merged tree $T_{n e w}$ into flash memory. Refer to [3] for more detail. Although the D-Forest requires merge operations, these operations incur a smaller number of page writes than applying the insert or delete operation repeatedly, as will be shown in Sect. 3.5.

Note that the same key can appear more than one B+tree in a D-Forest. If the same key appears in more than one $\mathrm{B}$ +-tree to be merged, we insert only the one in the lowest order tree (with or without a deletion mark) into $T_{\text {new }}$. Note that the lower the order of a tree, the more recently it has been constructed. We regard $T^{R}$ as the lowest order tree. Especially when $T^{R}$ and all trees in $D F$ are merged into only one single tree $T_{n e w}$ in $D F$, those keys that have deletion marks in their lowest level trees are not inserted into $T_{n e w}$. Note that in this case $T_{\text {new }}$ has only those keys that have no deletion marks.

\subsection{Search in D-Forest}

To find a key in a D-Forest, we first search $T^{R}$. If the key is not found in $T^{R}$, we search B+-trees stored in flash mem- ory in order of increasing order. That is, we search more recently constructed trees first. If the key is found with a deletion mark, we return Not found. According to the proposed merge strategy, the number of valid keys, i.e., nondeleted keys, in a D-Forest cannot fall below half the total number of keys in the D-Forest. Therefore, when the number of valid keys is $N$, the total number of keys in the DForest cannot exceed $2 N$. Since the number of keys in a tree $T_{k}$ is $O\left(2^{k}\right)$, the number of trees in the D-Forest for storing $2 N$ keys is $O(\log N)$. Therefore, the worst case time complexity of search in a D-Forest is $O\left((\log N)^{2}\right)$. Although this worst case time complexity is higher than that in a B+-tree, i.e., $O(\log N)$, read operations are very fast in flash memory.

\subsection{Concurrent Access to D-Forest}

During the merge operation of $T^{R}$ and $\left\{T_{0}, T_{1}, \ldots, T_{m-1}\right\}$, $T^{R}, T_{0}, T_{1}, \ldots, T_{m-1}$ cannot be updated. It can reduce concurrency of index access especially when a tree with a high order is involved in the merge operation. To address this problem, we use the following technique. First of all, note that $T^{R}, T_{0}, T_{1}, \ldots, T_{m-1}$ are never updated during the merge operation. They are invalidated only after the merge operation is completed. When the merge operation is started, we create an empty tree $T^{R^{\prime}}$ in RAM. The role of $T^{R^{\prime}}$ is essentially the same as that of $T^{R}$. That is, during the merge operation, if a key is inserted or deleted, we insert the key into $T^{R^{\prime}}$ instead of $T^{R}$. If a key is looked up, we first search $T^{R^{\prime}}$, then we search $T^{R}, T_{0}, T_{1}, \ldots, T_{m-1}$. When the merge operation is completed, $T^{R}$ is removed from RAM and $T^{R^{\prime}}$ becomes $T^{R}$. In this way, insertion, deletion, and lookup of keys can be concurrently performed during the merge operation. Note that $\left|T^{R^{\prime}}\right|$ can also reach its maximum allowed size during the merge operation. In this case, we can create another empty tree $T^{R^{\prime \prime}}$ in RAM.

\subsection{Analysis of D-Forest}

\subsubsection{The Number of Page Writes}

In this subsection, we compare the number of page writes in $\mathrm{D}$-Forest and the conventional $\mathrm{B}+$-tree. Let $N$ be the number of keys to be inserted into the index. We count the number of page writes occurring during the insertion of $N$ keys into an initially empty D-Forest and B+-tree, respectively. For simplicity, we assume that after the insertion of $N$ keys, all the trees in $D F$ are merged into one single tree $T_{L}$ such that $D F=\left\{T_{L}\right\}$. We also assume that all keys are unique. $B$ is the blocking factor, which represents the maximum number of entries in each $\mathrm{B}+$-tree page. $P$ is the size of a page in NAND flash memory.

When a key is inserted into a $\mathrm{B}+$-tree with $i$ keys, at least $\left\lceil\log _{B} i\right\rceil$ page writes are required since the height of the $\mathrm{B}+$-tree is $\left\lceil\log _{B} i\right\rceil$. If we ignore the number of page writes due to the node split operations, the total number of page writes $W_{\text {Btree }}$ for inserting $N$ keys into a $\mathrm{B}+$-tree is as follows: 


$$
\begin{aligned}
W_{\text {Btree }} & =\sum_{i=1}^{N-1}\left\lceil\log _{B} i\right\rceil \geq \log _{B}(N-1) ! \\
& \approx(N-1) \log _{B}(N-1)-(N-1)+\alpha
\end{aligned}
$$

where $\alpha=\frac{\log _{B}(N-1)}{2}+\frac{\log _{B}(2 \pi)}{2}[4]$.

In the D-Forest, $D F$ is constructed as follows: $\left\{T_{0}\right\} \rightarrow$ $\left\{T_{1}\right\} \rightarrow\left\{T_{0}, T_{1}\right\} \rightarrow\left\{T_{2}\right\} \rightarrow\left\{T_{0}, T_{2}\right\} \rightarrow\left\{T_{1}, T_{2}\right\} \rightarrow\left\{T_{0}, T_{1}, T_{2}\right\}$ $\rightarrow \ldots \rightarrow\left\{T_{0}, T_{1}, T_{2}, \ldots, T_{L-1}\right\} \rightarrow\left\{T_{L}\right\}$. Since each $T_{i}(0 \leq$ $i \leq L)$ is written into flash memory $2^{L-i}$ times and the number of pages required to store $T_{i}$ is $\left|T_{i}\right| / P=\left(2^{i} \cdot M\right) / P$, the total number of page writes $W_{D F}$ in the D-Forest is as follows:

$$
W_{D F}=\sum_{i=0}^{L} 2^{L-i} \cdot \frac{\left(2^{i} \cdot M\right)}{P}=(L+1) \cdot \frac{2^{L} \cdot M}{P}
$$

Recall that the number of keys in $T_{L}$ is $N$. Since the number of pages in a level $\left.i\left(1 \leq i \leq\left\lceil\log _{B} N\right)\right\rceil\right)$ of $T_{L}$ is $N / B^{i}$, the total number of pages in $T_{L}$ is $\left|T_{L}\right| / P=\left(2^{L} \cdot M\right) / P=$ $\sum_{i=1}^{\left\lceil\log _{B} N\right\rceil} N / B^{i}=(N-1) /(B-1)$. Thus, $L=\log _{2}(P / M \cdot(N-$ $1) /(B-1))$. Consequently, $W_{D F}$ can be expressed as follows:

$$
W_{D F}=\frac{(N-1)}{(B-1)} \cdot\left(\log _{2} \frac{(N-1)}{(B-1)}-\beta\right)
$$

where $\beta=\log _{2} \frac{M}{P}+1$. The ratio of $W_{B t r e e}$ to $W_{D F}$ is:

$$
\frac{W_{B T r e e}}{W_{D F}}=\frac{(B-1)}{\log _{2} B} \cdot \frac{\log _{2}(N-1)-\log _{2} B+\alpha}{\log _{2}(N-1)-\log _{2}(B-1)-\beta}
$$

Thus, when $N$ is sufficiently large, the ratio of $W_{\text {Btree }}$ to $W_{D F}$ approaches $(B-1) / \log _{2} B$. For example, when $B=$ $64,(B-1) / \log _{2} B=10.5$. This means that the number of page writes in the D-Forest is about ten times less than that in the conventional B+-tree. Therefore, in many practical applications, D-Forest can be effectively used to reduce the number of page writes.

\subsubsection{The Number of Pages}

In this subsection, we compare the number of pages required for storing $N$ keys in the conventional $\mathrm{B}+$-tree and $\mathrm{D}$-Forest. $B$ is the blocking factor and $P$ is the size of a page as defined above.

For a $\mathrm{B}+$-tree, the average storage utilization $S U$ is known to be about 0.7 [2]. Since the number of pages in a level $\left.i\left(1 \leq i \leq\left\lceil\log _{B} N\right)\right\rceil\right)$ of a $\mathrm{B}+$-tree is $N / B^{i}$ in case of maximizing storage utilization, the average number of pages for a B+-tree, $P_{\text {Btree }}$, is as follows:

$$
P_{\text {Btree }}=\frac{1}{S U} \cdot \sum_{i=1}^{\left\lceil\log _{B} N\right\rceil} N / B^{i} \approx \frac{10}{7} \cdot \frac{(N-1)}{(B-1)}
$$

For a D-Forest, the number of pages for $D F=\left\{T_{0}, T_{1}\right.$, $\left.\ldots, T_{K}\right\}$ is expressed as $\sum_{i=0}^{K}\left|T_{i}\right| / P=\sum_{i=0}^{K}\left(2^{i} \cdot M\right) / P$. Let $N_{i}$ be the number of keys in $T_{i}$. Since $\left|T_{i}\right| / P=\left(2^{i} \cdot M\right) / P=$ $\left(N_{i}-1\right) /(B-1)$ as described in the previous subsection, $N_{i}$ $=\left(2^{i} \cdot M\right) \cdot(B-1) / P+1 \approx\left(2^{i} \cdot M\right) \cdot(B-1) / P$. Note that the storage utilization of $T_{i}$ is always maximized in D-Forest, as described in Sect. 3.3. In the best case, where there is no key in $D F$ that has a deletion mark, the total number of keys in $D F$ is $N$. Since $N=\sum_{i=0}^{K} N_{i}=\sum_{i=0}^{K}\left(2^{i} \cdot M\right) \cdot(B-1) / P$, the number of pages for $D F$, denoted by $P_{D F(B e s t)}$, is as follows:

$$
P_{D F(\text { Best })}=\sum_{i=0}^{K} \frac{\left(2^{i} \cdot M\right)}{P}=\frac{N}{(B-1)}
$$

In the worst case, where the number of valid keys in $D F$ is only half the total number of keys in $D F$, the total number of keys in $D F$ is $2 N$. Since $2 N=\sum_{i=0}^{K} N_{i}=\sum_{i=0}^{K}\left(2^{i} \cdot M\right) \cdot(B-$ 1) $/ P$, the number of pages for $D F$, denoted by $P_{D F(\text { Worst })}$, is as follows:

$$
P_{D F(\text { Worst })}=\sum_{i=0}^{K} \frac{\left(2^{i} \cdot M\right)}{P}=\frac{2 N}{(B-1)}
$$

Therefore, when $N$ is sufficiently large, $P_{D F(\text { Best })} \leq P_{\text {Btree }} \leq$ $P_{D F(\text { Worst })}$.

\section{Performance Evaluation}

In order to evaluate the performance of D-Forest, we have implemented B+-tree and D-Forest on a NAND flash simulator with a page size of $4 \mathrm{~KB}$. We conducted trace-driven simulations using the following two types of traces. The size of a key was set to 16 bytes.

- Synthetic traces: We generated the following three traces using synthetic data.

1. Insert trace: 1,000,000 randomly generated records are inserted.

2. Delete trace: 500,000 records are randomly deleted after the insert trace is completed.

3. Lookup trace: 10,000,000 records are randomly retrieved according to the Pareto distribution after the insert trace is completed.

- Real traces: we used three traces received from [5] that contain B+-tree operations performed in the Reiser file system for executing the following tasks.

1. Kernel-compile trace: the Linux kernel source is untarred, compiled, cleaned, and removed.

2. Postmark trace: the postmark benchmark is executed, where lots of files are created and deleted.

3. MP3 trace: the storage of $8 \mathrm{~GB}$ is filled with MP3 files and then $50 \%$ of the files are deleted and copied again five times.

Because D-Forest maintains $T^{R}$ in RAM, we allocated the same amount of RAM to B+-tree for the read/write cache in order to make a fair comparison. $M$, the maximum size of $T^{R}$, was set to $16 \mathrm{~KB}$ in the experiments.

Figure 4 shows the performance of two indexes on the synthetic traces. Here, BT and DF represent the B+-tree and D-Forest, respectively. Figure 4 (a) shows the number of read and write operations performed in two indexes for 

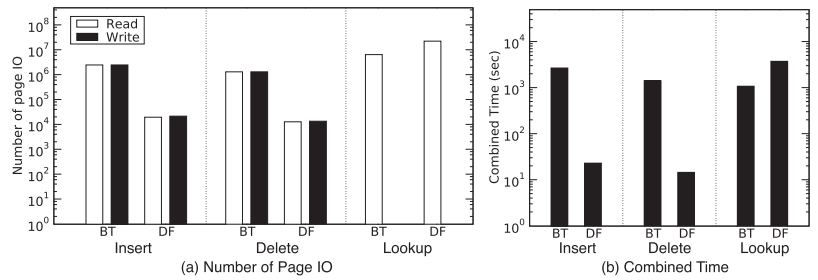

Fig. 4 Performance of D-Forest on the synthetic traces.
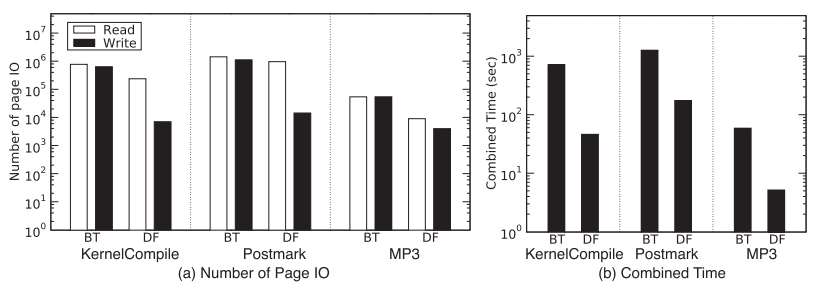

Fig. 5 Performance of D-Forest on the real traces.

each trace, while Fig. 4 (b) shows the total estimated time spent performing both read and write operations. The total estimated time $t_{e}$ is calculated as $t_{e}=165.6 \mu \mathrm{s} \times$ (the number of read operations) $+905.8 \mu \mathrm{s} \times$ (the number of write operations). The average read latency $(165.6 \mu s)$ and write latency $(905.8 \mu \mathrm{s})$ are taken from [6]. For the insert and delete traces, D-Forest shows a substantial improvement over B+tree in term of the number of write operations in even several orders of magnitude. (Note that the Y-axis is log scale.) However, as expected, D-Forest shows slightly worse performance than $\mathrm{B}+$-tree for the lookup trace.

Figure 5 shows the performance of two indexes on the real traces. Also in this case, D-Forest significantly outperforms B+-tree in terms of the number of write operations. Note that D-Forest results in even less number of read operations than $\mathrm{B}+$-tree for the real traces. This is because D-Forest keeps recently inserted keys in lower order trees.
In the real traces, recently inserted keys are quite frequently looked up. Since D-Forest searches the trees in order of increasing order, those keys can be quickly found in lower order trees.

\section{Conclusion}

We proposed an efficient index structure for NAND flash memory, called the Dynamic Forest (D-Forest). By coalescing many index updates into far fewer page writes, D-Forest can significantly reduce write operations, resulting in lower latency and lower energy consumption for NAND flashequipped devices. Through our experiments, we showed the performance advantage of D-Forest over the conventional B+-tree.

\section{Acknowledgments}

This research was supported by the Ministry of Knowledge Economy, Korea, under the Information Technology Research Center support program supervised by the Institute of Information Technology Advancement. (grant number IITA-2008-C1090-0801-0031), and Brain Korea 21 Project, the School of Information Technology, KAIST in 2009.

\section{References}

[1] E. Gal and S. Toledo, "Algorithms and data structures for flash memories," ACM Comput. Surv., vol.37, no.2, pp.138-163, 2005.

[2] D. Comer, "Ubiquitous B-tree," ACM Comput. Surv., vol.11, no.2, pp.121-137, 1979.

[3] S.-W. Kim, "On batch-constructing B+-trees: Algorithm and its performance evaluation," Inf. Sci., vol.144, pp.151-167, 2002.

[4] W. Feller, An Introduction to Probability Theory and Its Applications, vol.1, 3rd ed., Wiley, New York, 1968.

[5] CA Lab., KAIST, http://camars.kaist.ac.kr/, 2008.

[6] Samsung Elec., $2 \mathrm{G} \times 8$ Bit NAND Flash Memory (K9WAG08 U1A), 2006. 